\title{
Aberrometric, Keratometric, and Visual Outcomes After Trans-Epithelial Topography-Guided Phototherapeutic Keratectomy for the Treatment of Irregular Corneas
}

\author{
Antonio Cano-Ortiz (D) \\ Pablo Morales ${ }^{2}$ \\ Álvaro Sánchez-Ventosa' \\ Isabel Leiva-Gea ${ }^{3,4}$ \\ Alberto Membrillo',5 \\ Vasyl Druchkiv 6,7 \\ Timoteo González-Cruces' \\ José-María Sánchez- \\ González iD ${ }^{8}$ \\ Jaime Beltrán ${ }^{7}$ \\ Alberto Villarrubia' \\ 'Instituto de Oftalmología la Arruzafa, \\ Córdoba, Spain; ${ }^{2}$ Department of Cataract \\ and Refractive Surgery, Clínica Baviera, \\ Málaga, Spain; ${ }^{3}$ Hospital Regional \\ Universitario de Málaga, Málaga, Spain; \\ ${ }^{4}$ Istituto de Biomedicina de Málaga \\ (IBIMA), Málaga, Spain; ${ }^{5}$ Universidad de \\ Córdoba, Córdoba, Spain; ${ }^{6}$ University \\ Medical Center Hamburg Eppendorf, \\ Hamburg, Germany; ${ }^{7}$ Department of \\ Research \& Development, Clínica \\ Baviera, Valencia, Spain; ${ }^{8}$ Department of \\ Physics of Condensed Matter, Optics \\ Area University of Seville, Seville, Spain
}

Correspondence: Pablo Morales; Isabel Leiva-Gea

Málaga, Spain

Tel +34 669232767 ;

Tel +3467725405

Email pblmorales@hotmail.com;

isabeleiva@hotmail.com
Purpose: To assess the safety, aberrometric and keratometric changes, and stability of transepithelial topography-guided phototherapeutic keratectomy (TE-TG-PTK) with mitomycin $\mathrm{C}$ (MMC) using the ALLEGRO Topolyzer platform for the treatment of irregular astigmatism. Methods: This is a retrospective case series including 57 eyes that underwent TE-TG-PTK + MMC using the ALLEGRO Topolyzer platform for the treatment of irregular astigmatism. CDVA, manifest refraction (MR), keratometry readings, and aberrometry readings were analyzed at $1,3,6$, and 12 months.

Results: Causes of corneal irregularity included non-infectious leucoma ( $n=23)$, infectious leucoma $(n=7)$, adenoviral keratitis $(n=20)$, corneal haze $(n=2)$, post-penetrant keratoplasty (PKP) $(n=1)$, and others $(n=4)$. Overall, $76 \%$ of the eyes $(n=40)$ gained lines of vision; patients gained 1 , and 2 or more lines of vision in $76 \%$, and $38 \%$ of cases, respectively. Only 1 patient (2\%) lost 5 lines of vision. Mean preoperative CDVA (LogMAR) was $0.37 \pm 0.31$ and improved to $0.14 \pm 0.18(\mathrm{p}<0.001)$ at final follow-up (12 months). CDVA remained unchanged in 10 eyes $(21 \%)$. No significant changes were observed in mean keratometry (Kmean) and keratometric astigmatism readings. Regarding aberrometry, only changes in coma proved to be significant 6 months after surgery $(\mathrm{P}<0.01)$. No intraoperative/postoperative complications were reported.

Conclusion: At final follow-up, significant improvements were observed in CDVA and coma. TE-TG-PTK + MMC proved to be an effective and safe procedure for the treatment of corneal irregular astigmatism due to several causes.

Keywords: topography-guided phototherapeutic keratectomy, irregular astigmatism. laser refractive surgery, mitomycin $\mathrm{C}$

\section{Introduction}

Most cases of irregular corneal astigmatism include trauma, post-keratoplasty, corneal scars, keratoconus/ectasia, and post-refractive complications (ie, decentration, small ablation zones). The refractive management of these cases is challenging. As opposed to lower order aberrations (ie defocus and astigmatism) that can be corrected with spectacles, soft contact lenses, or several refractive surgery procedures, irregular corneas induce higher order aberrations, such as spherical aberration or coma, which are not suitable for correction by those means. Higher order aberrations affect the quality of vision of patients producing glare, halo, 
starbursts, and monocular diplopia, which affects deeply in their quality of life. Consequently, several methods have been used for the treatment in these cases. Rigid contact lens (CL) fitting usually is the first treatment attempted, also providing information about the patient's potential visual acuity. However, complications such as intolerance, peripheral neovascularization, infectious keratitis, and chronic allergic reactions, amongst others may limit its use.

Customized treatments have then been developed using either wavefront-guided (WFG) or topography-guided (TG) ablations to address these complex cases. WG treatments have shown very good results for the treatment of regular astigmatism or normal refractive surgery cases, however because the aberrations measured by the aberrometry devices consider the ones of the whole eye, results are usually not satisfactory in irregular corneas. ${ }^{1-7}$

Most cases of irregular astigmatism are secondary to corneal causes, and TG treatments have been used in these cases with satisfactory results. ${ }^{1-7,8-18,20,21}$ When the aberrations exist at the level of the cornea, the information provided by the topographer is then transferred to a laser platform, which subsequently plans a treatment based on the specific irregularity and higher order aberrations of that specific cornea. The regularizing procedure itself may induce some refractive shift that will further need to be corrected. $^{11,12,22}$ However, correcting errors on a regular surface offers much better outcomes and feasibility.

Several platforms have been developed to produce TG treatments. In the ALLEGRETTO WAVE Eye-Q system (WaveLight AG [Alcon Laboratories], Erlangen, Germany) (wavelength:193 nm, pulse duration: 12 nanoseconds, repetition rate: $400 \mathrm{~Hz}$ ) the surgeons can select treatment based on data generated by the Oculyzer or the Topolyzer. The former uses data from a Scheimpflug rotating camera that captures the eye either 25 or 50 times and obtains elevation information. As a result, numerous data is collected from the central cornea, and then is transferred to the laser platform via the Oculink software to plan the treatment. The Topolyzer uses a Placido-disk based system in order to measure 22,000 points to provide a curvature measurement of the cornea to be analyzed. This information is then transferred to the laser platform using the Topography Customized Ablation Treatment (T-CAT). ${ }^{8}$

The purpose of this study is to assess the safety, aberrometric and keratometric changes, and stability of transepithelial topography-guided phototherapeutic keratectomy
(TE-TG-PTK) using the ALLEGRO Topolyzer platform for the treatment of irregular astigmatism.

\section{Patients and Methods}

This is a retrospective case series including 57 eyes of 51 consecutive patients that underwent TE-TG-PTK using the ALLEGRO Topolyzer platform for the treatment of irregular astigmatism. Only patients with irregular astigmatism were included. Irregular astigmatism was considered as defined by Goggin et al, ${ }^{23}$ as the presence of best corrected distance visual acuity (CDVA) of one or more lines less than the visual acuity with a rigid CL/pinhole, coupled with the presence of topographic changes - either unequal slopes of hemimeridians along a single meridian, and/or hemimeridians of an equal slope but not aligned with each other.

All patients included completed at least 6-months of postoperative follow-up. Preoperative keratometry readings were obtained with the Topolyzer and then transferred to the T-CAT software in order to plan the surgical treatment. At least 8 images of good quality were included for the analysis. Treatment was programmed with an optical zone (O.Z) of $6.5 \mathrm{~mm}$ and a transition zone (T.Z) of $1.25 \mathrm{~mm}$. Patients with diagnosis of corneal opacities were assessed with AS-OCT Visante ${ }^{\mathrm{TM}}$ (Carl Zeiss, Germany) in order to estimate the depth of injury. In addition, over-refraction with a hard contact lens (CL) was performed in these patients in order to assess if either the corneal opacity or corneal irregularity was the main contributing factor in the patient's vision loss; if no improvement with the CL was seen, the corneal opacity was assumed as the predominant cause, and more ablation was programmed. In any case the treatment plan was calculated with a minimal residual stromal bed (RSB) thickness of at least 300 um. CDVA (LogMAR), Manifest Refraction (MR), keratometry readings, and aberrometry readings, were analyzed at 1, 3, 6, and 12 months. Preoperative and post-operative keratometry readings were obtained using 2 different topographers (either Visante Omni ${ }^{\circledR}$ or Pentacam ${ }^{\circledR}$ ). Data collected also included demographic characteristics, intraoperative, and postoperative complications. MR was performed using our standardized protocol. The ablation profile was centered on the corneal vertex determined by the topography. Vector analysis was not performed in this study, as this assumes a regular toric corneal surface opposed to the corneal profile of our patients. The corneal aberrations were obtained with either the Pentacam or Visante Omni 
topographer; each third and fourth order Zernike coefficient were analyzed during the entire study time span. Aberrometry indices that were analyzed included total High Order Aberration Root Mean Square (Total HOA RMS), total coma, and spherical aberration (SA). Considering that both devices (Pentacam and Visante Omni) use different technology in order to measure keratometry and aberrations, analyses were done separately for each parameter except total HOA RMS (Pentacam only). In cases of irregular astigmatism secondary to corneal transplantation (CT), TG-PTK was always performed at least 12 months after CT; and 6 months after the removal of the last sutures. Informed consent and permission to use patients' data for analysis and later publication was obtained from each patient prior to the procedure as part of our surgical protocol.

The study adhered to the tenets of the Declaration of Helsinki and was approved by the research ethics committee biomedical at Arruzafa hospital.

\section{Surgical Procedure}

All surgeries were performed by 2 surgeons (A.C.O and A.V. C). TE laser followed by TG-PTK with T-CAT software and the Allegretto Eye-Q 400-Hz excimer laser (Alcon Laboratories Inc.) was performed in all patients. In cases with previous diagnosis of corneal opacities, after the TEPTK treatment was completed, a conventional PTK procedure was performed prior to the TG-PTK in order to improve corneal clarity. Mitomycin C (MMC) $0.02 \%$ was instilled for 12 seconds after the laser was performed, and posteriorly rinsed away with balanced salt solution (BSS). A bandage contact lens was fit which remained in place until full epithelialization was confirmed during follow-ups. Moxifloxacin drops QID were used until the bandage contact lens was removed. Fluorometholone $0.1 \%$ eye drops were given for 2 months, with a tapered dosing schedule. Patients with previous history of herpetic keratitis were treated with oral Acyclovir $400 \mathrm{mg}$ BID for 2 months prior surgery and advised to continue for several months after the TE-TG-PTK.

\section{Statistical Analysis}

Microsoft Excel 2010 software (Microsoft Corporation) was used for data entry. To analyze changes in continuous variables random mixed regression method was used. ${ }^{25}$ This approach allows including all eyes even if some of them do not have measurements at all time points. This is especially advantageous in our case where we have dropouts at each postoperative time points. All P-values were adjusted for multiple comparisons with Tukey method. The analysis was performed with R (R Core Team, 2019). Analysis of variance (ANOVA) was performed to analyze changes over time. A P-value of $<0.05$ was considered significant.

\section{Results}

\section{Demographics}

57 eyes of 51 patients were included in the study. Mean age was $45.07 \pm 15.74$ years. Causes of corneal irregularity included: Non-infectious leucoma $(n=23)$, infectious leucoma $(n=7)$, adenoviral keratitis $(n=20)$, corneal haze $(n=2)$, postpenetrant keratoplasty (PKP) $(n=1)$, and others $(n=4)$ (Figure 1). 6 patients had bilateral affection; 5 of those patients (10 eyes) had adenoviral conjunctivitis; 1 patient had iron deposits.

\section{Safety}

52 eyes were analyzed at 12 months follow-up. Overall, 76\% of the eyes $(n=40)$ gained lines of vision; patients gained 1 , and 2 or more lines of vision in $76 \%$, and $38 \%$ of cases, respectively. Only 1 patient (2\%) lost 5 lines of vision. CDVA remained unchanged in 10 eyes $(21 \%)$ at final followup (Figure 2). No intra/postoperative complications were noticed during the entire follow-up period time.

\section{Corrected Distance Visual Acuity}

There was an overall improvement in CDVA (LogMAR). Mean preoperative CDVA was $0.37 \pm 0.31$, and improved to $0.14 \pm 0.18(\mathrm{P}<0.01)$ at final follow-up (12 months). CDVA improvement proved to be statistically significant between preoperative 3 , and 6 months $(\mathrm{P}<0.01)$. There was a trend towards improvement from 6 months to 12 months; however, these changes did not reach statistical significance $(\mathrm{P}=0.13)$ (Figure 3).

\section{Causes of Corneal Irregularity}

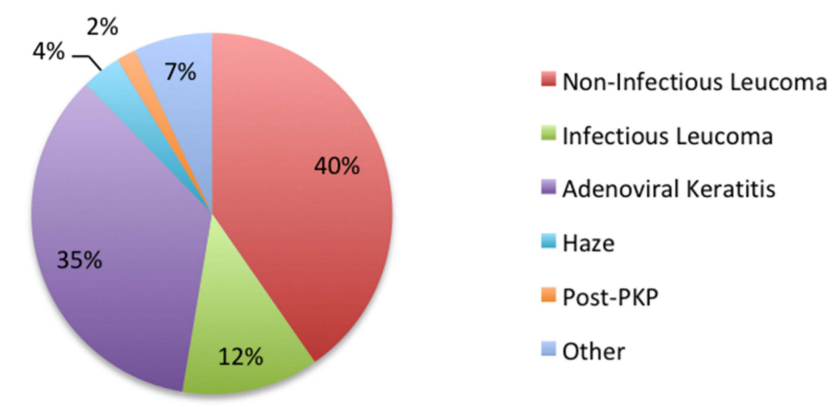

Figure I Causes of corneal irregularity. $n=57$ eyes. 6 cases were binocular. 


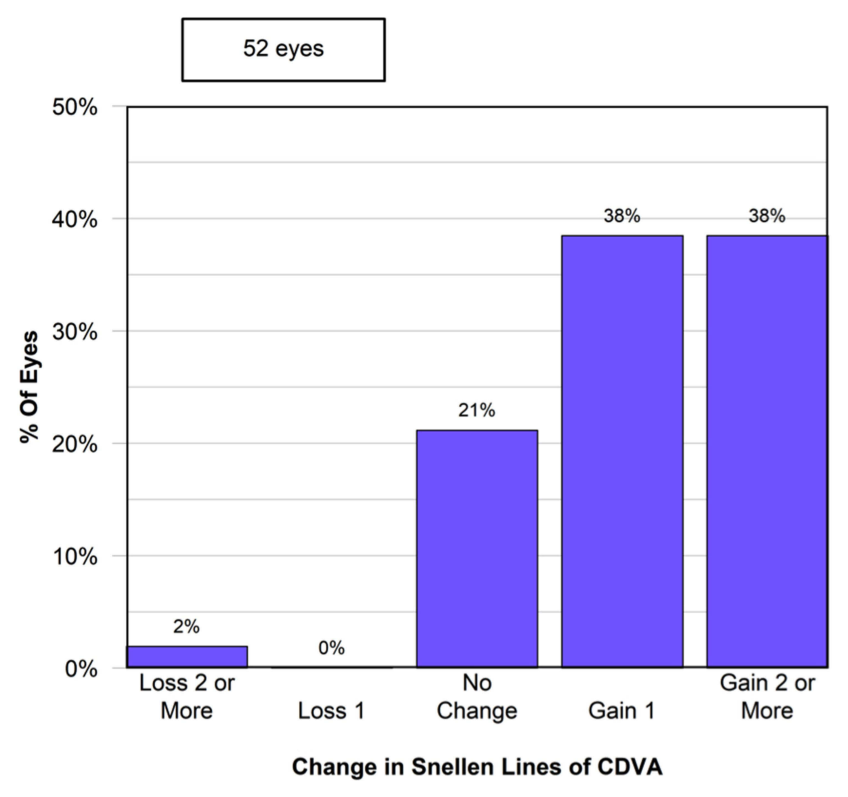

Figure 2 Safety. Changes in Snellen lines of CDVA at final follow-up (12 months). $n=52$.

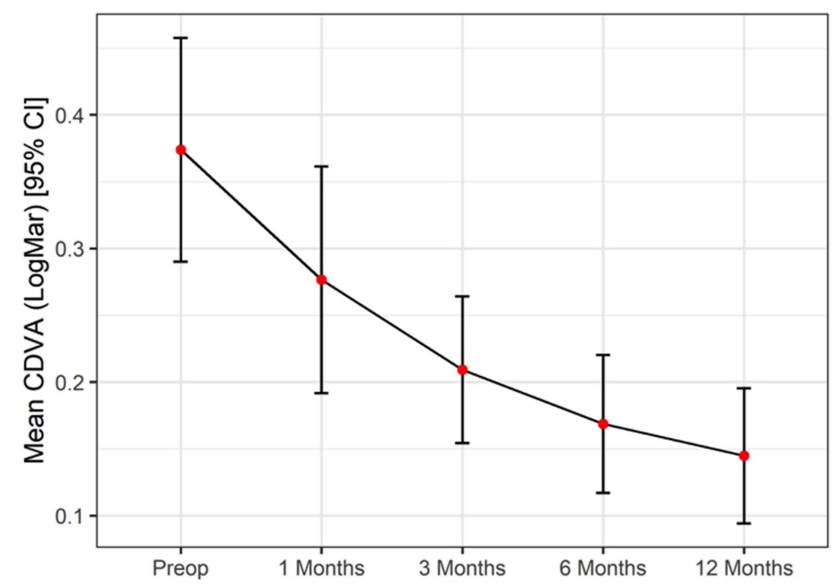

Figure 3 Improvement in CDVA (LogMAR) was significant at 3, and 6 months after surgery. CDVA stabilized after 6 months.

\section{Topographic and Aberrometric Analysis}

Overall, mean Keratometry (Kmean) measurements showed an increasing and significant trend from 43.53 $\pm 2.31 \mathrm{D}$ to $44.71 \pm 2.84 \mathrm{D}$ and $44.58 \pm 3.00 \mathrm{D}$ at 1 , and 3 months after surgery, respectively $(\mathrm{P}<0.01)$. The figures dropped close to baseline levels 6 months after the surgery (44.15 $\mathrm{D} \pm 2.78)$. There were insufficient data to analyze the 12 months endpoint. Figures measured with both devices show a similar pattern, although changes in the pentacam group were significant only 1 month after surgery $(\mathrm{P}=041)$. (Figure 4).
Mean preoperative corneal astigmatism was 2.4 D ( \pm 1.9$)$. Overall, no significant changes were seen at 6 , and 12 months $(\mathrm{P}=0.79$, and $\mathrm{P}=0.87$, respectively). No significant differences in the measurements from both devices were observed (Figure 5).

Aberrometry measurements studied included coma, SA, and Total HOAs RMS. Overall, significant changes were only seen in coma 6 months, and 12 months after the surgery $(\mathrm{P}<0.01)$. However, no statistically significant changes were observed between 6 and 12 months $(\mathrm{P}=0.94)$. In addition, no significant changes were observed neither in Total HOAs RMS nor SA during the entire follow-up period. A similar pattern was observed with either device. Total HOAs RMS were only acquired with pentacam. (Table 1; Figures 6 and 7.

\section{Discussion}

Many studies have demonstrated the effectiveness in TGtreatments for the treatment of corneal irregular astigmatism $^{2-6,8,10-14,17-21}$ with most of these being done in patients with irregular astigmatism secondary to keratoplasty, RK or post-refractive decentered/small zone ablations. Lains et $\mathrm{al}^{20}$ reported a retrospective case series of 31 eyes with irregular astigmatism after PKP treated with TG-PRK. At the last follow-up visit, $74.2 \%$ of the eyes $(n=23)$ gained CDVA lines and a gain of UDVA was also observed in $96.8 \%$ $(\mathrm{n}=30)$. This was accompanied by an improvement of MR without significant postoperative complications. Six cases (19.4\%) presented haze, which resolved without visual sequelae, and only 1 case with recurrent herpetic keratitis. Similarly, Bandeira e Silva et $\mathrm{al}^{18}$ reported significant improvements ( $80 \%$ of patients) in the BCVA of 15 postkeratoplasty eyes after TG-PRK. Additionally, twelve months postoperatively the lines of vision increased, and astigmatism, corneal best-fit sphere, mean keratometry, corneal thickness, corneal total RMS, and corneal SA decreased. In another retrospective series by Sorkin et al, ${ }^{9} 34$ eyes were treated with TG-PRK for post-PKP irregular astigmatism; this was followed by additional surface ablation in case of residual refractive error. In their study, mean CDVA also improved significantly: 1 line or more in $54.2 \%$, and 3 lines or more in $16.7 \%$ of the analyzed eyes. Improvement in CDVA is a good indicator of corneal regularization. In addition, objective aberrometry indices, such as total RMS, HOA RMS, defocus, coma, trefoil, and spherical aberration, also showed significant improvement. This study emphasizes the 


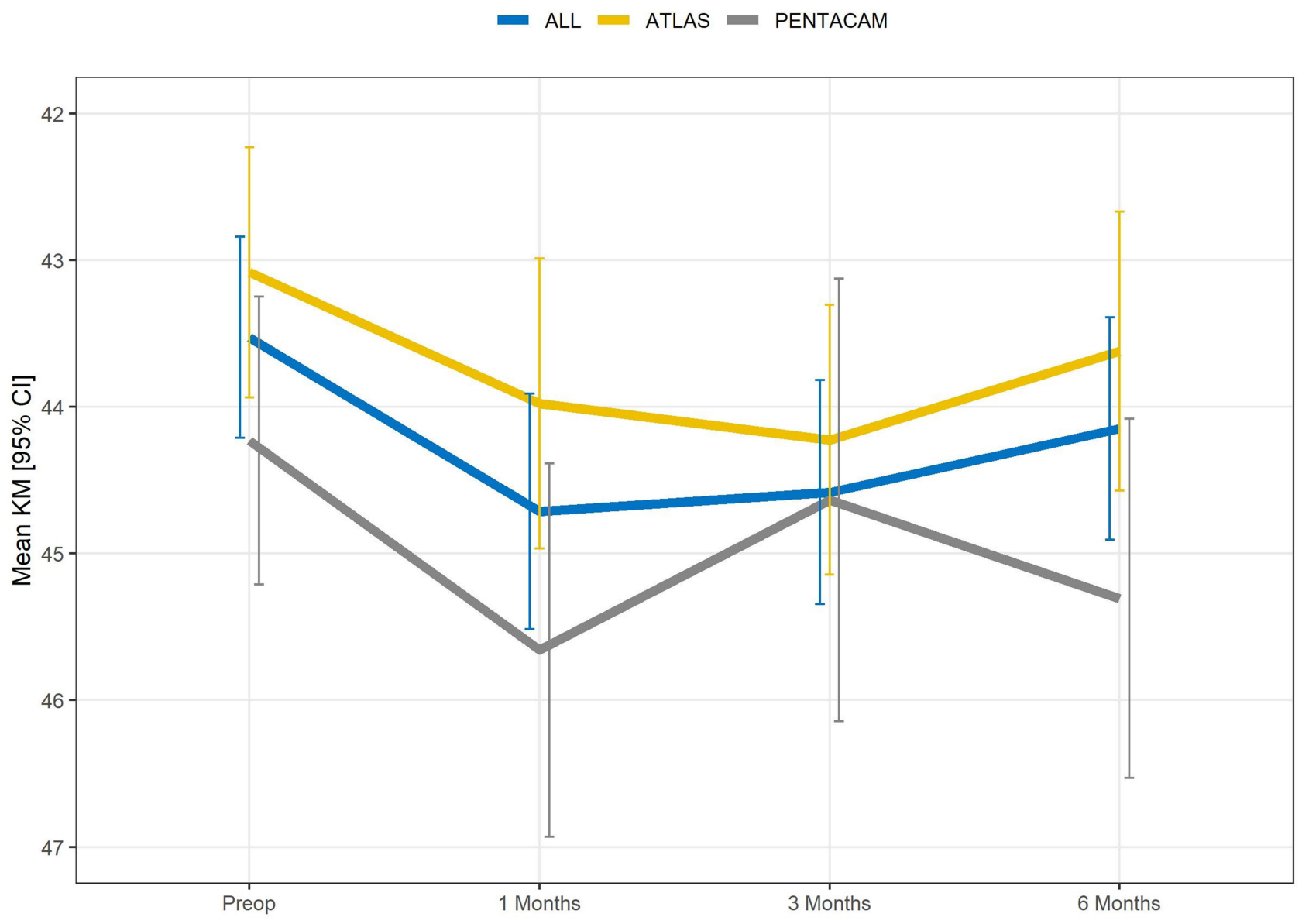

Figure 4 Kmean changes at I, 3, 6, and 12 months. Changes proved to be significant up-to 3 months after surgery. Measurements performed with different devices are shown in different colors.

need of patients to be counseled regarding the possibility of a secondary refractive procedure. As mentioned before, regularization of the surface by TG-ablations could increase the postoperative refractive error. In the largest retrospective published case series to date (54 eyes), and with the most long-term follow-up (mean 31 months), TG-PRK after PKP proved to have good long-term efficacy and safety, resulting in significant UDVA, refractive, and keratometric improvement. ${ }^{11}$ This study also stresses the importance of long-term follow-up, as regression occurred up to 4 years after treatment. They concluded that high levels of ametropia with astigmatism up to $7 \mathrm{D}$ can be effectively treated with a single ablation, and that even a partial treatment can improve the refractive error and irregular astigmatism, thus improving patient's quality of life, thus patients being able to function well with mild corrections or even without needing glasses or CL.

In our study, noteworthy changes in CDVA were also observed. CDVA improved in $76 \%$ of the eyes included; moreover, up to 10 lines of improvement were observed in 1 patient, and overall, $76 \%$ of the eyes $(n=40)$ gained lines of vision; patients gained 1, and 2 or more lines of vision in $76 \%$, and $38 \%$ of cases, respectively. This is of paramount importance, as some patients were able to avoid (or at least delay) a further corneal transplant procedure. We believe that the surgical protocol applied in our cohort played a major role for the considerable improvement in CDVA observed. Considering that $91 \%$ of the total eyes included in our study had opacity-related corneal irregularities, using AS-OCT Visante ${ }^{\mathrm{TM}}$ in order to assess the actual depth of the opacity, and consequently trying to remove most part of the opacity, and finally using the TCAT treatment to manage the residual irregularity was a plausible reason for the striking CDVA improvement. Sloan et al, ${ }^{21}$ also studied OCT-guided TE-PTK for cases of irregular astigmatism secondary to anterior corneal scarring. In their retrospective study, 64 eyes were included, showing promising results in BCVA, UCVA, 

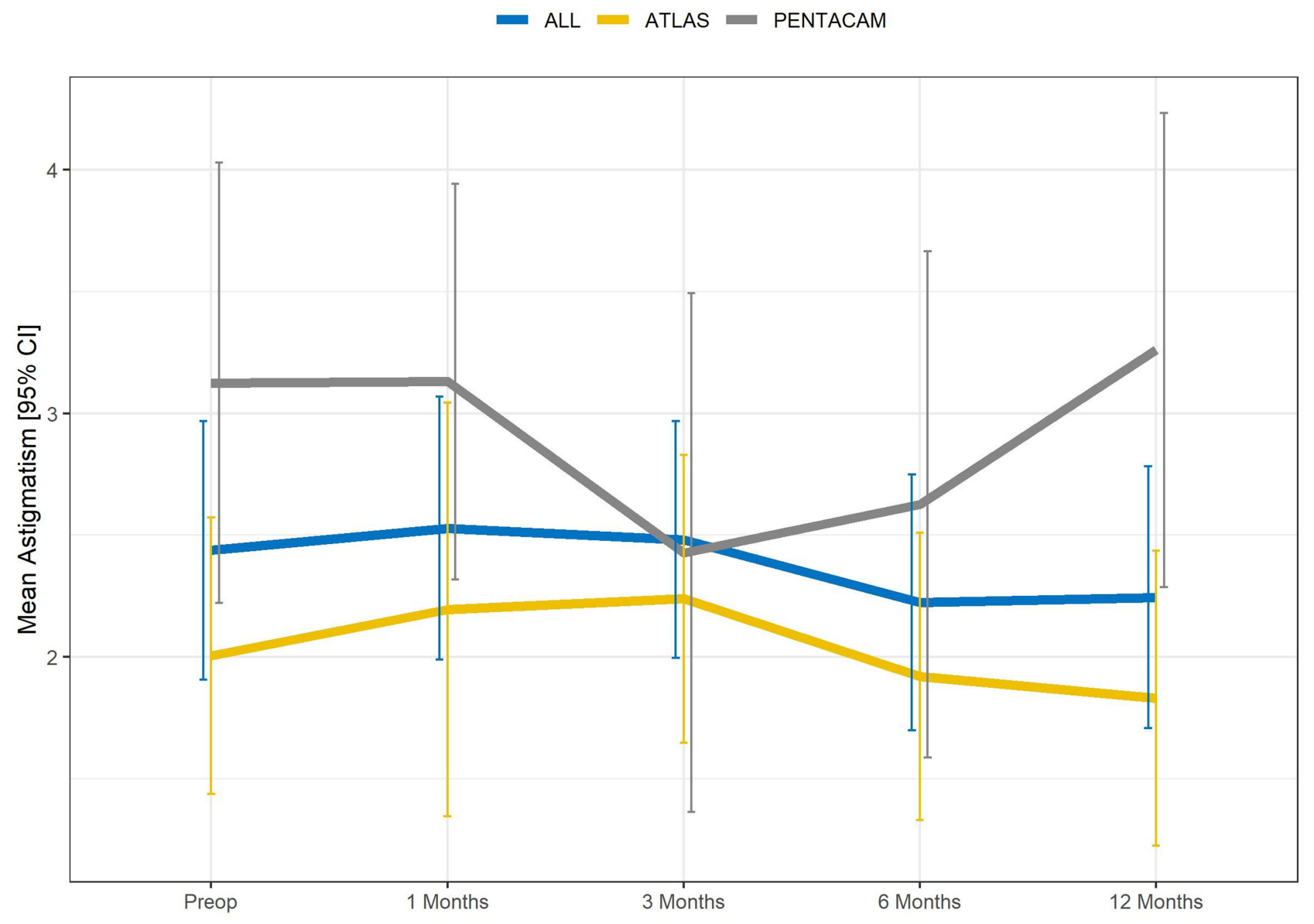

Figure 5 Mean corneal astigmatism measured with both Pentacam and Atlas 9000 topographers. Measurements performed with different devices are shown.

corneal astigmatism, surface asymmetry index, surface regularity, and projected visual acuity; however, in his study no customized treatments were performed, remaining uncertain if a customized treatment could have provided even better outcomes. In another study by Spadea et al, ${ }^{14} 26$ eyes with superficial corneal opacities were treated using a TG-PRK algorithm; the estimated ablation was calculated according to the depth of the opacities. In their study $76.9 \%$, and $23.1 \%$ of cases, gained 1 , and 2 or more lines, respectively. Corneal opacities, however, were assessed biomicroscopically using digitalized slit lamp pictures. We consider that when available AS-OCT is crucial for an appropriate, and accurate diagnosis, and treatment planning in these cases.

In terms of safety, only 1 patient (2\%) experienced a significant decrease in CDVA (5 lines) due to irregular astigmatism induced postoperatively. This patient had previous diagnosis of non-infectious leucoma unfortunately. It is possible that dense scarred corneas produce unpredictable responses leading to a worsening of the irregularity; unfortunately this patient lost to follow-up 6 months after the procedure. Further observations need to be done in order to assess which patterns of corneal scarring will react better to a laser corneal ablation.

TG-treatments have also been used to manage postrefractive surgery-related complications. In a case series of 73 eyes by Reinstein et al, ${ }^{5}$ TG-PRK proved to be effective in enlarging the optical zone, recentering the optical zone, and reducing higher order aberrations. In their study, the optical zone centration improved by $63 \%$, and optical zone diameter was increased by $11 \%$; subsequently there was a significant reduction in higher order aberrations, with a $53 \%$ reduction in coma and $44 \%$ reduction in spherical aberration. This also translated in a significant improvement in subjective night vision disturbances in $93 \%$ of the cases, and $55 \%$ of patients reporting complete resolution of symptoms.

Topographic astigmatism was also assessed in our study. There was an initial increase 1 month postoperatively in topographic astigmatism from $2.44 \pm 1.90 \mathrm{D}$ to 
Table I Changes in Corneal Aberrations Measured with Both Devices. Parameters Estimated with Mixed Regression Model. Parameters are Given as Estimate (Standard Error)

\begin{tabular}{|c|c|c|c|c|c|c|c|}
\hline & \multicolumn{2}{|l|}{ All } & \multicolumn{2}{|l|}{ ATLAS } & \multicolumn{3}{|c|}{ PENTACAM } \\
\hline & $\begin{array}{l}\text { Coma } \\
\text { (um) }\end{array}$ & $\begin{array}{l}\text { Spherical } \\
\text { Aberration } \\
\text { (um) }\end{array}$ & $\begin{array}{l}\text { Coma } \\
\text { (um) }\end{array}$ & $\begin{array}{l}\text { Spherical } \\
\text { Aberration } \\
\text { (um) }\end{array}$ & $\begin{array}{l}\text { Coma } \\
\text { (um) }\end{array}$ & $\begin{array}{l}\text { Spherical } \\
\text { Aberration } \\
\text { (um) }\end{array}$ & RMS \\
\hline & -1 & -2 & -3 & -4 & -5 & -6 & -7 \\
\hline Preop & $\begin{array}{r}1.247 \\
-0.14\end{array}$ & $\begin{array}{l}0.360 \\
-0.04\end{array}$ & $\begin{array}{l}0.928 \\
-0.13\end{array}$ & $\begin{array}{l}0.377 \\
-0.06\end{array}$ & $\begin{array}{r}1.652 \\
-0.27\end{array}$ & $\begin{array}{l}0.333 \\
-0.06\end{array}$ & $\begin{array}{l}2.307 \\
-0.35\end{array}$ \\
\hline Change (Preop-6 months) & $\begin{array}{l}-0.449 * * * . \\
0.12\end{array}$ & $\begin{array}{l}0.03 \\
-0.05\end{array}$ & $\begin{array}{l}-0.329 * * \\
-0.13\end{array}$ & $\begin{array}{l}-0.06 \\
-0.08\end{array}$ & $\begin{array}{l}-0.620^{* *} \\
-0.22\end{array}$ & $\begin{array}{l}0.10 \\
-0.06\end{array}$ & $\begin{array}{l}-0.41 \\
-0.29\end{array}$ \\
\hline Change (Preop- 12 months) & $\begin{array}{l}-0.435^{* * *} \\
-0.12\end{array}$ & $\begin{array}{l}-0.05 \\
-0.05\end{array}$ & $\begin{array}{l}-0.327^{* *} \\
-0.13\end{array}$ & $\begin{array}{l}-0.08 \\
-0.08\end{array}$ & $\begin{array}{l}-0.612^{* *} \\
-0.23\end{array}$ & $\begin{array}{l}-0.02 \\
-0.08\end{array}$ & $\begin{array}{l}-0.602 * \\
-0.30\end{array}$ \\
\hline SD between & 0.65 & 0.15 & 0.27 & 0.07 & 0.85 & 0.17 & 0.95 \\
\hline SD within & 0.77 & 0.24 & 0.62 & 0.28 & 0.95 & 0.19 & 1.34 \\
\hline ANOVA. P value & 0.003 & 0.362 & 0.056 & 0.610 & 0.034 & 0.219 & 0.069 \\
\hline Observations & 100 & 99 & 56 & 56 & 44 & 43 & 64 \\
\hline
\end{tabular}

Notes: ${ }^{*}<<0.1 ; * *_{p}<0.05 ; * * * p<0.01$.

$2.52 \pm 1.77 \mathrm{D}$; this was followed by a decreasing trend 3 months postoperatively, reaching lower-than baseline levels at final follow-up (2.24 $\pm 2.04 \mathrm{D})$. Nonetheless, none of these changes proved to be significant. Moreover, the mean preoperative astigmatism of our cohort was not very high $2.44 \mathrm{D} \pm 1.90$ and this could also have played some part in the final outcome, as the effect of TG-treatments is more noticeable with larger astigmatisms treated. Kmean readings followed a similar pattern, with an initial surge 1 month postoperatively from $44.63 \pm 2.31 \mathrm{D}$ to $44.58 \pm 2.94$ $\mathrm{D}$, and a subsequent decrease until final follow-up (44.12 $\pm 2.31 \mathrm{D}$ ); again this changes were not significant. Epithelial hyperplasia has been observed in the early stages of the healing process in these cases, and may have played a role in this initial increment observed in both keratometry and astigmatism readings. ${ }^{14}$ In addition, the increase in keratometry readings may also be explained due to the aspherical ablation profile of the TG-treatment: In order to compensate the energy loss from the oblique corneal peripheral pulses, the energy provided to the periphery must be higher than the energy applied to the central cornea (perpendicular pulses); in consequence the central corneal curvature increases. Further studies with longer follow-up and larger cohorts of patients are needed in order to assess if these changes are of clinical interest.
Only few groups have studied aberrometric changes with TG-PRK for irregular astigmatism after keratoplasty. ${ }^{15-19}$ In our study, not only post-keratoplasty patients were included. In our cohort there was a significant decrease in coma from $1.25 \pm 1.17$ preoperative to $0.84 \pm 0.71$ at 6 months $(\mathrm{P}<0.01)$; these changes remained stable at final follow-up. In addition, 12 months postoperatively there was a mean reduction of $0.602 \pm 0.3$ um, and $0.327 \pm 0.13$ um in the mean total HOA RMS and $\mathrm{SA}$, respectively; although these changes were not significant ( $\mathrm{P}=0.19$, and $\mathrm{P}=0,62$, respectively), we can only assume that the regularization of the corneal surface is responsible for the massive improvement seen in CDVA. Due to the small sample size, we could not assess if there were causes of corneal irregularity in which TG-treatment would result in a better outcome. Bandeira e Silva et al successfully treated highly aberrated eyes with the highest mean preoperative RMS in the literature $(9.60 \mathrm{um})$. They obtained a mean reduction of 1.52 um in total RMS. ${ }^{18}$ Rajan et al, ${ }^{15}$ and Camellin et $\mathrm{al}^{16}$ also reported decreases in total coma and SA. Ghoreishi et al, ${ }^{8}$ studied TE-TGPRK in 22 eyes with irregular astigmatism post-radial keratotomy (RK). They demonstrated the procedure to be effective, safe, and predictable up-to 1-year follow-up. Furthermore, HOAs (mean SA and mean coma) 


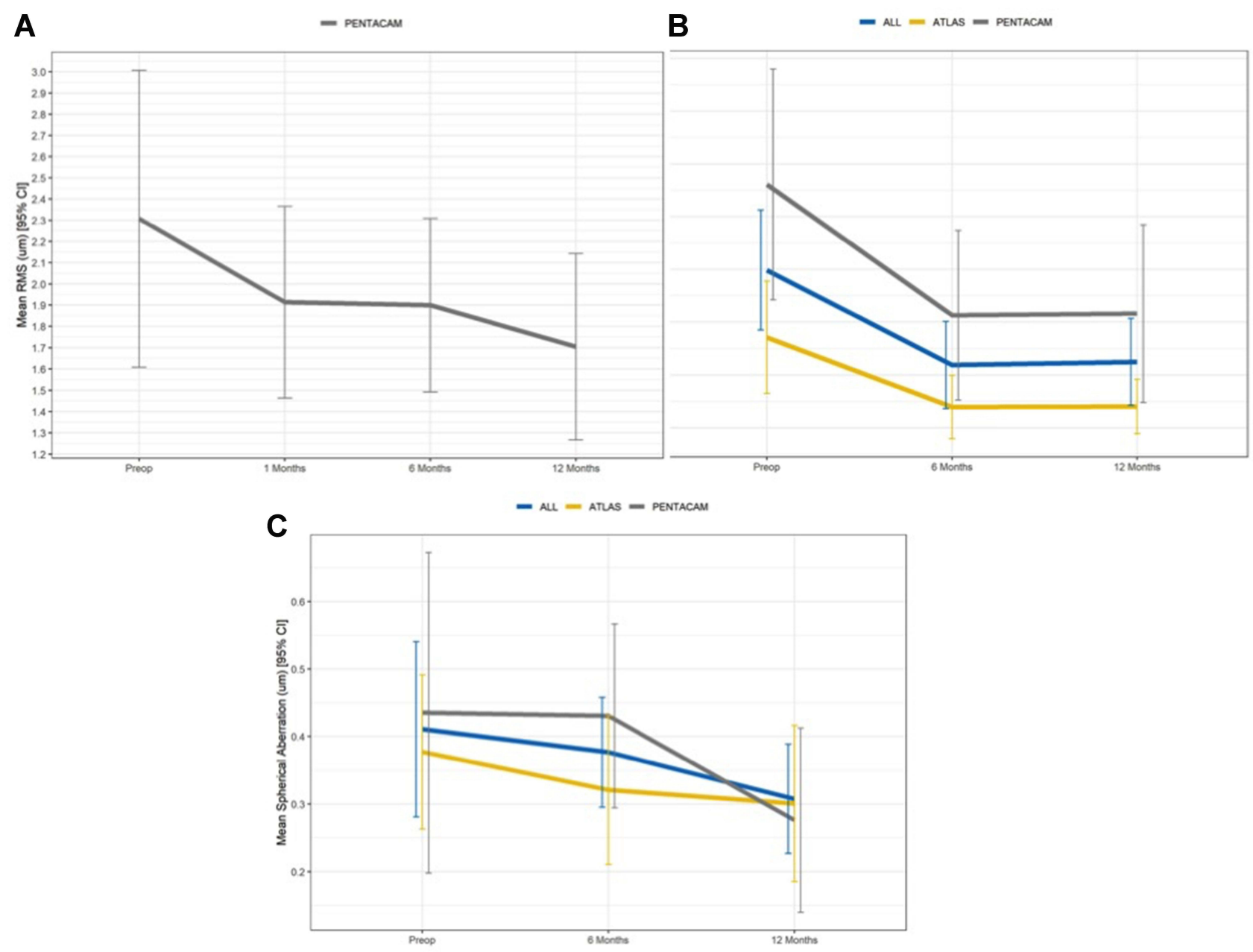

Figure 6 Changes in corneal aberrations during the entire follow-up period. (A) No significant changes were observed in Total HOA. (B) Overall, changes in coma were significant at 6 months after surgery $(\mathrm{P}<0.0 \mathrm{I})$, and remained unchanged after. $(\mathbf{C})$ No significant changes were observed in SA. Measurements performed with different devices are depicted.

significantly reduced at the 6-month visit. Sorkin et al ${ }^{10}$ found a statistically significant improvement in the analyzed aberration parameters measured including total RMS, HOA RMS, defocus, coma, trefoil, and spherical aberration in 13 eyes.

Reinstein et al, considered the compensatory epithelial remodeling effect over masking stromal irregularities from the corneal surface during TE-PTK. ${ }^{15}$ They concluded that customized treatments (either TG or WG) may not provide an adequate treatment option and may even potentially worsen the irregularity. The effectiveness of TE-PTK will depend on the proportion of epithelial masking due to remodeling, related to the rate of change of curvature of the stromal surface.

In conclusion, overall customized treatments prove to be an effective and safe procedure for the treatment of corneal irregular astigmatism. In our series we noticed significant improvement in CDVA, and coma. These changes proved to be more pronounced at 6 months, and then remained stable up to final Follow-up (1 year). Over-refraction using preoperative hard CL fitting also provided information regarding the amount of tissue needed to be ablated: if vision improved after CL fitting, irregularity was believed to be the main contributing factor to the low CDVA; on the other hand, if no improvement in CDVA was observed, the corneal opacity was assumed to play a major role and more tissue was required to be ablated in order to improve vision. We believe that the surgical protocol applied in our study: TE-PTK using the epithelial layer as a masking agent, in addition to preoperative AS-OCT, and over-refraction with hard CL in cases of corneal opacities, contributed to the good CDVA and safety results observed. Nevertheless, TG- 


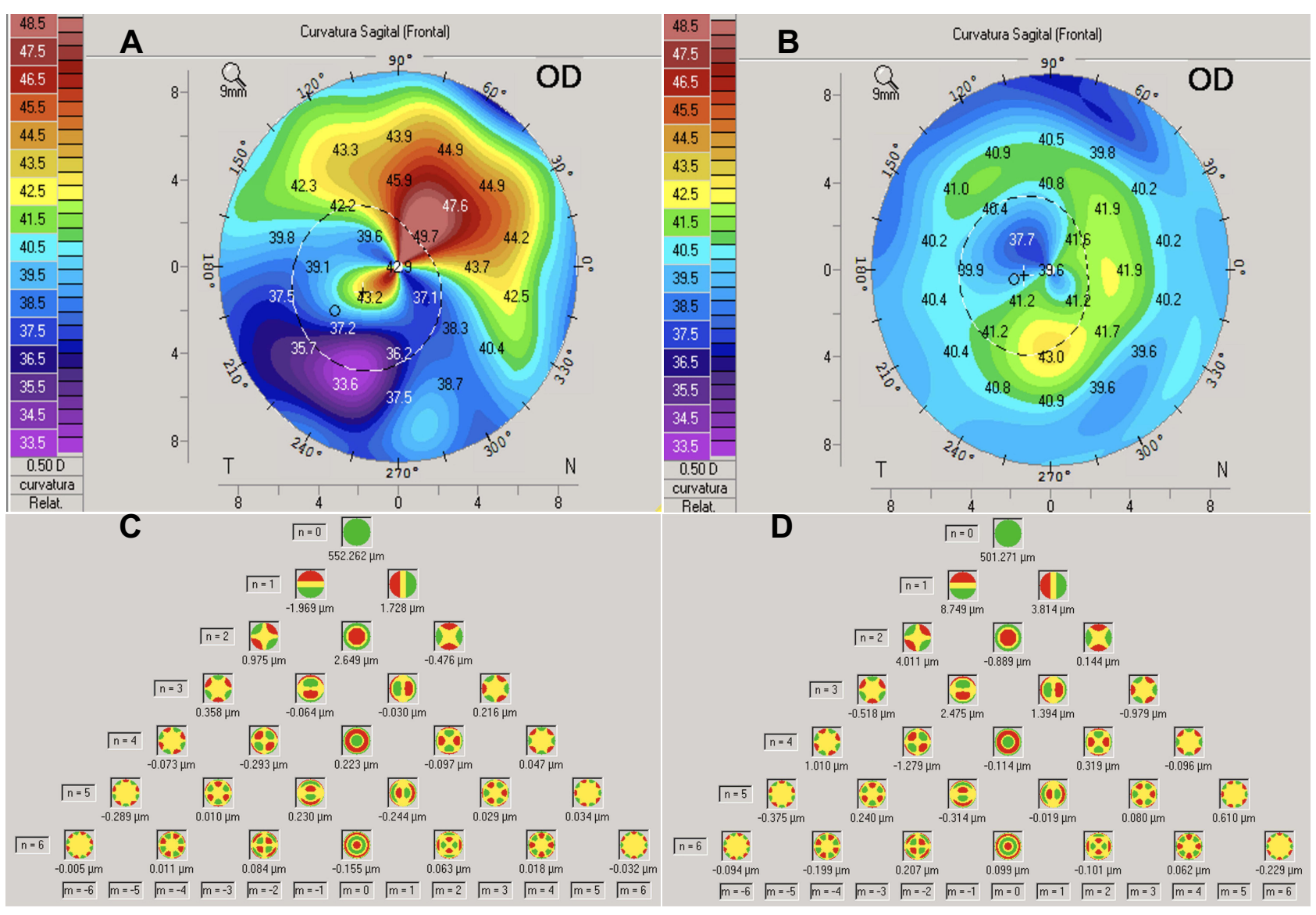

Figure 7 Preoperative and postoperative topographic, and aberrometric changes after TE-TG-PTK at 12 months follow-up. Sagittal maps (A, B) show substantial regularization of the corneal surface: Kmax decreased from $44.0 \mathrm{D}$ preoperatively (A) to $4 \mathrm{I} .5 \mathrm{D}$ postoperative (B) without a major decrease in Kmean (4I.3 D (A) to $40.8 \mathrm{D}(\mathbf{B})$ ). Zernike analysis (C, D) also shows considerable changes in total coma with figures decreasing from 2.84 um preoperatively (C) to 0.070 um (sum of the mean root square) at final follow-up (D). This patient had previous diagnosis of diffuse corneal leucomas secondary to adenoviral keratoconjunctivitis, and gained 6 lines of Snellen CDVA (from LogMAR 1.92 to -0.1 ) at final follow-up.

treatment planning might be inaccurate because of epithelial hyperplasia and stromal remodeling, masking irregularities that may probably lead to inaccurate ablation profiles and residual refractive errors. Consequently, counseling of these patients on a long-term follow-up and a subsequent refractive procedure is crucial for patient's satisfaction.

\section{Disclosure}

The authors declare no potential conflicts of interest in this work.

\section{References}

1. Reinstein DZ, Archer TJ, Couch D, Schroeder E, Wottke M. A new night vision disturbances parameter and contrast sensitivity as indicators of success in wavefront-guided enhancement. J Refract Surg. 2005;21(5):S535-S540. doi:10.3928/1081-597X-20050901-23

2. Lin DT, Holland S, Tan JC, Moloney G. Clinical results of topography-based customized ablations in highly aberrated eyes and keratoconus/ectasia with cross-linking. J Refract Surg. 2012;28(11 Suppl):S841-S848. doi:10.3928/1081597X-20121005-06
3. Holland S, Lin DT. Topographically directed PRK for irregular astigmatism following penetrating keratoplasty. Poster presented at: American Academy of Ophthalmology Annual Meeting, November 11-14; 2006; Las Vegas, NV.

4. Mrochen M, Jankov M, Bueeler M, Seiler T. Correlation between corneal and total wavefront aberrations in myopic eyes. $J$ Refract Surg. 2003;19(2):104-112. doi:10.3928/1081-597X-20030301-04

5. Reinstein DZ, Archer TJ, Carp GI, et al. Incidence and outcomes of optical zone enlargement and recentration after previous myopic LASIK by topography-guided custom ablation. $J$ Refract Surg. 2018;34(2):121-130. doi:10.3928/1081597X-20171215-01

6. Toda I, Yamamoto T, Ito M, Hori-Komai Y, Tsubota K. Topography-guided ablation for treatment of patients with irregular astigmatism. J Refract Surg. 2007;23(2):118-125. doi:10.3928/1081-597X-20070201-03

7. Mrochen M, Krueger RR, Bueeler M, Seiler T. Aberration-sensing and wavefront-guided laser in situ keratomileusis: management of decentered ablation. $J$ Refract Surg. 2002;18(4):418-429. doi:10.3928/1081-597X-20020701-01

8. Ghoreishi M, Peyman A, Koosha N, Golabchi K, Pourazizi M. Topography-guided transepithelial photorefractive keratectomy to correct irregular refractive errors after radial keratotomy. J Cataract Refract Surg. 2018;44(3):274-279. doi:10.1016/j.jcrs.2017.12.015

9. Sorkin N, Einan-Lifshitz A, Abelson S, et al. Stepwise guided photorefractive keratectomy in treatment of irregular astigmatism after penetrating keratoplasty and deep anterior lamellar keratoplasty. Cornea. 2017;36:1308-1315. doi:10.1097/ICO.0000000000001359 
10. Bizrah M, Lin DT, Babili A, Wirth MA, Samuel Arba-Mosquera S, Holland SP. Topography-guided photorefractive keratectomy for postkeratoplasty astigmatism: long-term outcomes. Cornea. 2020;1:1-10.

11. Alió JL, Belda JI, Osman AA, Shalaby AM. Topography-guided laser in situ keratomileusis (TOPOLINK) to correct irregular astigmatism after previous refractive surgery. J Refract Surg. 2003;19(5):516-527. doi:10.3928/1081-597X-20030901-06

12. Jankov MR 2nd, Panagopoulou SI, Tsiklis N, Hajitanasis GC, Aslanides IM, Pallikaris IG. Topography-guided treatment of irregular astigmatism with the wavelight excimer laser. J Refract Surg. 2006;22:335-344.

13. Spadea L, Bianco G, Balestrazzi E. Topographically guided excimer laser photorefractive keratectomy to treat superficial corneal opacities. Ophthalmology. 2004;111(3):458-462. doi:10.1016/j. ophtha.2003.06.002

14. Reinstein DZ, Archer TJ, Gobbe M. Improved effectiveness of transepithelial PTK versus topography-guided ablation for stromal irregularities masked by epithelial compensation. J Refract Surg. 2013;29 (8):526-533. doi:10.3928/1081597X-20130719-02

15. Rajan MS, O'Brart DP, Patel P, Falcon MG, Marshall J. Topographyguided customized laser-assisted subepithelial keratectomy for the treatment of postkeratoplasty astigmatism. J Cataract Refract Surg. 2006;32(6):949-957. doi:10.1016/j.jcrs.2006.02.036

16. Camellin M, Arba Mosquera S. Simultaneous aspheric wavefront-guided transepithelial photorefractive keratectomy and phototherapeutic keratectomy to correct aberrations and refractive errors after corneal surgery. J Cataract Refract Surg. 2010;36 (7):1173-1180. doi:10.1016/j.jcrs.2010.01.024

17. De Rosa G, Boccia R, Santamaria C, Fabbozzi L, De Rosa L, Lanza M. Customized photorefractive keratectomy to correct high ametropia after penetrating keratoplasty: a pilot study. J Optom. 2015;8(3):174-179. doi:10.1016/j.optom.2013.12.002
18. Bandeira E, Silva F, Hazarbassanov RM, Martines E, Güell JL, Hofling-Lima AL. Visual outcomes and aberrometric changes with topography-guided photorefractive keratectomy treatment of irregular astigmatism after penetrating keratoplasty. Cornea. 2018;37 (3):283-289. doi:10.1097/ICO.0000000000001474

19. Sorkin N, Kreimei M, Einan-Lifshitz A, et al. Wavefront-guided photorefractive keratectomy in the treatment of high astigmatism following keratoplasty. Cornea. 2019;38(3):285-289. doi:10.1097/ ICO.0000000000001830

20. Laíns I, Rosa AM, Guerra M, et al. Irregular astigmatism after corneal transplantation - efficacy and safety of topography-guided treatment. Cornea. 2016;35(1):30-36. doi:10.1097/ ICO.0000000000000647

21. Sloan WR, Matulich J, Ryan BR. Long-term outcomes of optical coherence tomography-guided transepithelial phototherapeutic keratectomy for the treatment of anterior corneal scarring. $\mathrm{Br}$ $J$ Ophthalmol. 2014;98(12):1702-1706. doi:10.1136/bjophthalmol2014-305366

22. Hull CC. Loss of resolution in a corneal topography system. Graefes Arch Clin Exp Ophthalmol. 1999;237(10):800-805. doi:10.1007/ s004170050315

23. Goggin M, Alpins N, Schmid LM. Management of irregular astigmatism. Curr Opin Ophthalmol. 2000;11(4):260-266. doi:10.1097/00055735-200008000-00007

24. Pinheiro JC, Bates D. Mixed-Effects Models in S and S-Plus. Springer Science \& Business Media; 2006.

25. $\mathrm{R}$ Core Team. $R: A$ Language and Environment for Statistical Computing. Vienna, Austria: $\mathrm{R}$ Foundation for Statistical Computing; 2019. Available from: https://www.R-project.org/. Accessed August 30, 2021.
Clinical Ophthalmology

\section{Publish your work in this journal}

Clinical Ophthalmology is an international, peer-reviewed journal covering all subspecialties within ophthalmology. Key topics include: Optometry; Visual science; Pharmacology and drug therapy in eye diseases; Basic Sciences; Primary and Secondary eye care; Patient Safety and Quality of Care Improvements. This journal is indexed on PubMed

\section{Dovepress}

Central and CAS, and is the official journal of The Society of Clinical Ophthalmology (SCO). The manuscript management system is completely online and includes a very quick and fair peer-review system, which is all easy to use. Visit http://www.dovepress.com/ testimonials.php to read real quotes from published authors. 